\title{
Carnets
}

Revue électronique d'études françaises de l'APEF

Deuxième série - $20 \mid 2020$

Imaginaire(s) du Voyage

\section{Voyage(s) au bout de la mine chez Émile Zola et Fernando Namora}

\section{Odete Jubilado}

\section{(2) OpenEdition}

1 Journals

\section{Édition électronique}

URL : http://journals.openedition.org/carnets/12417

DOI : 10.4000/carnets. 12417

ISSN : 1646-7698

Éditeur

APEF

\section{Référence électronique}

Odete Jubilado, «Voyage(s) au bout de la mine chez Émile Zola et Fernando Namora », Carnets [En ligne], Deuxième série - 20 | 2020, mis en ligne le 30 novembre 2020, consulté le 19 avril 2021. URL : http://journals.openedition.org/carnets/12417; DOI : https://doi.org/10.4000/carnets. 12417

Ce document a été généré automatiquement le 19 avril 2021

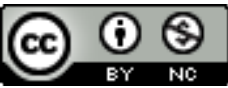

Carnets est mis à disposition selon les termes de la licence Creative Commons - Atribution - Pas d'utilisation commerciale 4.0 International. 


\title{
Voyage(s) au bout de la mine chez Émile Zola et Fernando Namora
}

\author{
Odete Jubilado
}

Dantes, a montanha era pacífica e farta. Dava pão,
vinho, castanhas e lã (...). E eis novamente a
montanha visitada pela cobiça do minério (...). A
montanha, mesmo assim, não quis acreditar. Pão,
vinho, castanhas e lã. Nunca daquelas fragas
tinha saído mais. Mas o Manco, o pastor, achou
um seixo muito pesado com laivos negros. Foi
vendê-lo ao senhor Williams. Quarenta e cinco
mil réis ! Alto ! Os fraguedos, afinal, valiam. E a
montanha deixou de dar pão, vinho, castanhas e
lã para dar minério (Torga, 1941 :171, 173).

1 Nous nous proposons d'esquisser une réflexion comparative axée sur deux voyage(s) au centre de la terre, à partir de l'analyse de Germinal (1885) d'Émile Zola (1840-1902) et de Minas de San Francisco (1946) de Fernando Namora (1919-1989) dont on vient de fêter en 2019 le centenaire de la naissance. Malgré leurs différences notre hypothèse de lecture vise justement à analyser ce voyage dans les profondeurs de la terre dont les deux romans se font les porte-voix.

Pour ce faire, nous baliserons notre réflexion en deux volets complémentaires. Dans un premier volet, il s'agira d'analyser ce voyage au bout de la mine à partir de sa topographie. Dans le deuxième volet, nous réfléchirons aux implications idéologiques de ce voyage sur l'homme comme membre d'une communauté, dans la mesure où, comme Henri Lefebvre (2000) le montre dans sa Production de l'Espace, l'espace, pour autant qu'il soit occupé par des êtres humains, est toujours déjà socialement construit, selon les codes et les valeurs économiques, idéologiques, religieux et politiques de la société des références.

3 Pour commencer, nous orienterons notre réflexion vers notre premier volet axé sur ce voyage au centre de la terre qui est proposé au lecteur. 


\section{Voyage au centre de la terre}

L'espace de vie d'Étienne Lantier et de João Simão se constitue d'un environnement matériel et technique : la mine; d'un environnement naturel : le paysage ; et enfin, d'un environnement intersubjectif dans le face à face avec l'homme. Ce sont les passages de l'un à l'autre qui assurent la fusion de ces espaces durant le voyage vers et dans la mine.

Dans Figures du Discours, l'Abbé Fontanier a considéré la description comme « une figure $\mathrm{du}$ discours autre que les tropes (...) une des figures de pensées » (1968: 420). Selon lui, la description comprend différentes espèces, entre lesquelles, la topographie ${ }^{1}$ : «Tout ce que nous dirons de la description en général, c'est qu'elle consiste à exposer un objet aux yeux, et à le faire connaître par le détail de toutes les circonstances les plus intéressantes » (Fontanier, 1968: 420). Selon cette classification, la description de la mine relèverait de la «topographie », c'est-à-dire "de la description qui a pour objet un lieu quelconque tel qu'un vallon, une montagne, une plaine, une ville, un village, une maison (...), une grotte, un jardin (...), une forêt, etc. (...) » (Fontanier, $1968: 420$ ).

Or, c'est justement cette topographie de la mine qui est dressée à l'égard du lecteur dès les premières pages des deux romans qui l'orientent vers le voyage d'un personnage en direction de la mine et vers les successifs voyages dans la mine (Zola, 1978 : 90-91). Ces voyages se manifestent à travers la présence d'un lexique du voyage au long des deux romans comme l'attestent les expressions suivantes: "Pendant un voyage; chaque voyage » (Zola,1978: 90-91); «o viajante senta-se num penedo» (Namora, 2018: 21); «O viajante sorriu» (Namora, 2018: 25). En fait, la description de l'arrivée d'un étranger (Étienne Lantier ou João Simão) dans ce milieu qui lui est inconnu permet de faire découvrir l'univers de la mine au lecteur. Effectivement, c'est à travers la description du paysage et du déplacement de ces deux personnages que ce milieu, particulièrement inhumain et hostile de la mine et des mineurs, est donné à voir et à imaginer au lecteur.

7 En réalité, la description des lieux est effectuée en mouvement au fur et à mesure de leur découverte par un personnage, ce qui la rend dynamique, proche du cinéma et du travelling puisque la caméra se déplace avec le personnage nous faisant connaître ce paysage minier. Tout le pays minier est ainsi décrit à travers les allées et venues des personnages qui l'arpentent, le paysage étant appréhendé selon différents points de vue et selon plusieurs circonstances. Nous avons, par exemple, celui du narrateur omniscient qui s'efface souvent derrière son personnage et adopte son point de vue. C'est précisément le cas d'Étienne Lantier qui voit et appréhende le monde dès la première page du roman étant donné que l'omniscience du narrateur est relayée par son regard. Ainsi, Étienne se déplace dans un paysage hostile et fantastique dont il a une perception floue et inquiétante. Ce paysage initial fera d'ailleurs l'objet de plusieurs déplacements d'Étienne au long du roman ce qui permettra au narrateur de multiplier les points de vue sur ce paysage initial.

Aussi bien Germinal que Minas de San Francisco exhibent l'influence que le paysage exerce sur l'homme. Un paysage particulièrement inhumain et hostile qui flagelle l'homme dans sa quête héroïque et constante à la recherche d'une subsistance quotidienne. En effet, dès leurs incipit (Zola, 1978: 49-50), (Namora, 2018: 19-20), les deux romans informent le lecteur sur les circonstances, les personnages, nouant avec lui un pacte de lecture. C'est justement sur la marche nocturne d'un homme/voyageur qui traverse un 
paysage hostile et désolateur en direction au Nord que les deux incipit s'ouvrent. De ce fait, dès les premières lignes des deux romans, l'attention du lecteur est captée par la présence de ces deux hommes pauvres, sans travail et égarés dans les ténèbres qui s'opposent à la force démesurée d'un paysage inhumain et effrayant, où une nature hostile fustige l'homme.

Aux ténèbres du paysage font place les « ténèbres de la mine » (Zola, 1978 : 94) décrites comme « buraco negro » chez Namora (2018 : 31). Ces ténèbres coïncident dans les deux romans avec les conditions de travail dans la mine auxquelles nous accédons, chez Zola, quand Étienne Lantier descend dans la mine pour la première fois (Zola, 1978 : 85-98). En effet, la descente d'Étienne permet au narrateur omniscient de peindre les conditions difficiles de travail des mineurs dans la mine à travers la description de l'activité des quatre haveurs de l'équipe: Maheu, Chaval, Zacharie et Levaque. Une description qui s'organise autour des quatre éléments (terre, feu, air et eau) qui semblent s'acharner contre l'homme. C'est le cas, par exemple, de Maheu qui se retrouve au centre de la terre, dans la taille où travaillent les haveurs à plus de 700 mètres sous la surface.

C'est précisément ce milieu étrange, hostile et étouffant où travaille l'homme muni d'une lampe de mineur, « un point rougeâtre » (Zola, 1978 : 87), dans l'obscurité absolue qui chauffe et brûle, que décrit le narrateur. Ce dernier évoque "Cette nuit sans astres» (Zola, 1978: 92) et étouffante à laquelle les yeux essayent de s'adapter : «Un instant il resta les regards noyés au fond des ténèbres de la mine; et à cette profondeur, sous le poids et l'étouffement de la terre » (Zola, 1978: 94). Dans ce milieu nocif à l'homme règne une température élevée qui «montait jusqu'à trente-cinq degrés ", « l'air ne circulait pas » et « l'étouffement à la longue devenait mortel » (Zola, 1978 : 86). En outre, ce milieu se caractérise par « les poussières volantes du charbon » (Zola, 1978 : 87), la présence de " gaz » et l'humidité. Dans cette chaleur, Maheu « (...) est trempé, couvert de sueur lui-même, fumant d'une chaude buée de lessive » (Zola, 1978: 86). C'est précisément à travers Maheu que le narrateur décrit aussi les conditions de travail difficiles du mineur, insistant sur l'aspect irréel de la mine qui apparaît associé à l'obscurité sur laquelle l'incipit insistait déjà : «Et il semblait que les ténèbres fussent d'un noir inconnu ${ }^{2}$, épaissi par les poussières volantes du charbon, alourdi par des gaz qui pesaient sur les yeux » (Zola, 1978: 87). C'est précisément cette couleur noire associée à la mort qui rythme ce paysage minier au long du roman.

11 La souffrance des mineurs rappelle au lecteur celle des damnés de l'Enfer, la mine ressemblant à une fournaise située dans les profondeurs de la terre, ce qui révèle l'inconfort humain de ce lieu inerte et, par conséquent, la contradiction entre les véritables besoins de l'homme et sa présence dans la mine. C'est pourquoi, les souffrances de Maheu s'apparentent à une véritable damnation où les quatre éléments de l'espace de la mine semblent s'unir pour le torturer. Rien n'échappe à cette torture, même un objet familier et utile comme la lampe du mineur sert ce propos : «Et cette lampe, qui chauffait son crâne, achevait de lui brûler le sang" (Zola, 1978: 86). Nous retrouverons d'ailleurs cet objet aussi chez Namora à travers la présence du « gancho dos lampiões " (Namora, 2018: 50). La violence des expressions choisies souligne bien l'horreur et le tourment infernal auquel sont soumis Maheu et les autres mineurs. C'est justement à travers la description des conditions de travail des mineurs à la fin du XIX ${ }^{\mathrm{e}}$ siècle que Germinal se constituera aussi comme un précieux document. En fait, ce rôle documentaire attribué au roman naturaliste conduira Zola à l'étude du milieu des 
mineurs, révélant leurs pulsions héréditaires, ainsi en va-t-il d'Étienne Lantier. Toutefois, le lecteur parviendra à la conclusion que, malgré l'hérédité et le milieu, certains personnages comme Étienne révèlent une volonté individuelle. Dès lors, Étienne décide, par exemple, de ne pas tuer Chaval chez Rasseneur. La souffrance de ces damnés innocents sera à la base de leur révolte ultérieure.

Chez Namora la description, basée sur une connaissance effective et concrète de cette réalité ainsi que sur le contact direct avec ces populations, imprime aussi à la fiction ${ }^{3}$ un caractère documentaire, la transformant en un document historique, économique, social, voire ethnographique précieux. Le lecteur retrouve ainsi les "terres maigres", les conditions climatiques défavorables, qui conduisent à de bonnes ou à de mauvaises années agricoles, telles que les hivers rigoureux avec leur "hálito gelado das madrugadas » (Namora, 2018 : 19-20) ou encore les étés torrides avec un soleil étouffant et la poussière des chemins où le « centeio, mirrado da seca, verga-se ao cálido sopro das serranias »; « crosta ressequida »; « Depois, o sol. Um sol duro, imóvel, crestando o oiro das searas "; «O pó dos caminhos amontoava-se em rios de secura » (Namora, $2018: 19-20)$.

La non-appartenance de la terre à l'agriculteur, comme le souligne cet extrait: «Conhece uma terra que não lhe pertence » (Namora, $2018: 19-20)$, avec des propriétés en bail, ce qui implique l'obéissance à un propriétaire, en plus des conditions économiques difficiles, n'empêchent cependant pas l'amour et la nostalgie de la terre. En l'occurrence parce que ces populations rurales sont pauvres parce que les champs qu'elles cultivent sont pauvres. La lutte pour la survie se manifeste aussi à travers d'autres formes distinctes telles que le travail dans la mine et la contrebande du wolfram ${ }^{4}$ dans les mines à travers António, l'un des principaux contrebandiers « candongueiros » de wolfram (Namora,2018: 96). La mine contrôle ainsi tous ceux qui essaient de voler cet «or noir»: «Um escombreiro que terminara o seu turno foi apalpado pelo guarda, desde as axilas às virilhas (...). - Se assim não fosse, traziam o volfrâmio agarrado às virilhas (...). San Francisco é uma ladroeira " (Namora, 2018 : 63-64).

Ce groupe de "camponeses feitos mineiros" (Namora, 2018: 93) est composé de "pessoas desenraizadas que não querem confessar a si próprios que ali não é seu mundo. Prendem-se à mina, e ao que ela lhes oferece, com fúria e um secreto temor » (Namora, 2018 : 54). Cependant, ils n'ont pas le choix puisque « já não confiam na terra que lhe sangrou a esperança » (Namora, 2018: 19). La mine représente donc le rêve d'une vie meilleure pour le peuple qui vit une illusion collective, San Francisco se transformant en un

formigueiro humano ${ }^{5}$ que crescia de todos os lados, estripando o solo com enxadas e picaretas, às vezes com as unhas, fazendo aflorar camadas de terra ensebada ou xistosa (...). Vinham sôfregos de uma vida nova (...). Por isso não minguam os rios de gente que desaguam na mina, gente de todas as idades e de todos os ofícios. (Namora, $2018: 47-48$ )

En fait, les deux romans construisent une image négative de la mine en tant qu'environnement souterrain artificiel construit par l'homme, dénonçant les conditions très difficiles de son habitabilité où l'air et la lumière font défaut. Le travail à la mine métamorphose les hommes en animaux chez Zola, Maheu est comparé à « un puceron pris entre deux feuillets d'un livre sous la menace d'un aplatissement complet » (Zola,1978: 86) ; Lydie pousse sa berline, "raidissant ses bras et jambes d'insecte, pareille à une maigre fourmi noire en lutte contre un fardeau trop lourd", 
tandis que, chez Namora, San Francisco devient "une fourmilière humaine » (Zola, $1978: 88,104)$.

La métaphore animale traverse les deux romans et les mineurs sont souvent bestialisés étant comparés aux fourmis qui symbolisent le travail mais aussi par l'analogie physique avec cet animal parce que les fourmis vivent dans et de la terre, construisant leurs propres galeries. Comme la fourmi, le mineur se caractérise par la couleur noire du charbon : "Ils étaient déjà noirs de charbon, enduits d'une poussière fine que la sueur délayait, faisait couler en ruisseaux et en mares (...). [Catherine] était noire, la face poudrée de charbon fin» (Zola, 1978: 88, 94). De plus, les deux mines sont assimilées à un monstre. Chez Namora, la mine est "um mostrengo em marcha » (Namora, 2018 : 33), « um formigueiro de corpos e de monstros de aço » (Namora, 2018 : 93). Chez Zola, la mine de "la fosse du Voreux » est comparée à une «bête goulue » (Zola, 1978: 52) à la respiration forte qui dévore les hommes qui y plongent. La métaphore du Voreux traverse le roman, c'est comme si la mine était le labyrinthe d'un monstre, une sorte de Minotaure, pour lequel les mineurs ne sont qu'un sacrifice humain en son honneur.

Dans les deux romans, l'esquisse du portrait des mineurs ne se limite ni à la description du paysage dans lequel la mine est insérée, ni à sa description souterraine avec ses galeries, ni même à la description de leurs conditions de travail. Le lecteur a également accès aux problèmes de santé des mineurs causés par l'environnement minier.

Tandis que Zola s'est documenté sur les maladies typiques des mineurs, Fernando Namora décrit sous le regard clinique du médecin les conséquences de cette descente aux enfers du mineur. Chez Zola, le personnage Bonnemort symbolise les effets néfastes de la mine : «sa face plate, d'une pâleur livide, maculé de taches bleuâtres (...). Il a les jambes raidies par des rhumatismes, tousse et crache»(Zola, 1978: 55, 51). L'expression «il cracha noir» (Zola, 1978: 57) rythme le texte et dénonce l'une des maladies typiques des mineurs: la silicose pulmonaire. De même chez Namora, cette maladie est présente comme l'atteste l'extrait suivant: "Anda um ror de gente a escarrar negro" (Namora, 2018: 37) en raison de la contamination de l'air par la poussière, des maladies respiratoires chroniques s'accumulent et consomment progressivement des mineurs anesthésiés qui ne remarquent même plus qu'ils travaillent dans un environnement suffocant comme dans l'extrait qui suit :

Os olhos dilatam-se para se adaptarem ao halo demasiado do carboneto. $\mathrm{O}$ hálito que sopra do extremo da galeria ou se escapa das paredes, que gemem um suor lustroso, vem infiltrar-se na carne, adensa-se nos brônquios. Os músculos estremecem, sacudidos pela súbita maleita (...). Os pulmões quase se habituam a essa asfixia, como os ouvidos já não escutam o ressoar cavernoso que, a espaços, se transforma num trovão. (Namora, $2018: 49$ )

O suor, a respiração, a poeira fina, fundem-se num granizo mole que se aninha nas máscaras, que se condensa nos alvéolos dos pulmões. Alguns marteleiros só enfiam a máscara quando a tosse convulsiva abre os músculos do peito ; mas jogam-na fora pouco depois com os olhos esbugalhados de sufocação. (Namora, $2018: 52$ )

À la surface, le scénario ne s'améliore pas pour les mineurs qui remontent abasourdis, détruisant leur seul atout précieux la santé, comme l'illustre la description de leurs corps malades: "as articulações parecem ter perdido o jogo de movimentos e os ventres ardem » (Namora, 2018 : 306). Le diagnostic clinique est en outre corroboré par le rosaire des maladies répertoriées par le médecin qui se déplace tous les jeudis à San Francisco pour observer les malades qui souffrent: "Quase todos se queixam de 
maleitas, dores no peito, catarrais (...), dedos esmagados, braços feridos »; « mineiros intoxicados, reumáticos do frio húmido das galerias" (Namora,2018: 185). À cette description des maladies s'ajoute la caractérisation de certains personnages tels que «le machiniste tuberculeux» (Namora,2018: 202) ou Almeida dont les «pulmões conhecem o tufo (...). o tufo condensa-se nas máscaras, corrói os pulmões, é impossível respirar com essa mudança a sorver o que resta de ar puro » (Namora, $2018: 57,37)$.

La destruction progressive du mineur et son épuisement se répercutent dans la "serenidade exausta da paisagem " (Namora, $2018: 202)$ dont la mine a également volé la vitalité. Comme Maheu, cultivant ses légumes et oubliant quelques instants le monde minéral de la mine pour profiter des bienfaits du monde végétal où règne la verdure, l'air et la lumière, João Simão, qui a toujours travaillé la terre, a le rêve tellurique de posséder un lopin de terre et d'avoir sa famille près de lui. C'est pourquoi il propose à Almeida de s'occuper gratuitement de son jardin comme l'illustre cet extrait : « Eu podia tratar cá da hortazinha (...). Irá zelar a horta como se lhe pertencesse (...). João Simão sonha com a sua beirada e com a vinda da sua família para junto de si » (Namora, $2018: 88,90)$. Soulignons l'utilisation du diminutif « hortazinha » qui révèle au lecteur l'affection qu'il porte à la terre, ce sentiment d'appartenance qu'il ne sentira jamais en relation à la mine. C'est un personnage qui vit sans cesse inquiet et qui interroge constamment la réalité qui l'entoure et qu'il ne comprend pas. Il ne s'est jamais adapté à l'âpreté de la mine :

Ainda não se libertou da sensação de que vive num esquife subterrâneo, de que por cima da sua cabeça há montanhas de solo, um mar de céu - e tudo se passa como se terra e céu se apoiassem sobre os seus ombros. Um peso que lhe esmaga o peito. (Namora, $2018: 50$ )

21 En réalité, seule la concrétisation de ce rêve tellurique mène João Simão à travailler à la mine :

João Simão (...) sonhava com uma horta, uma beirada, um pedaço a que chamasse seu, alguma coisa que fosse carne da sua carne, como nos tempos em que um homem, mesmo depois de morto, prolongava a sua existência nas árvores e na terra que os filhos lhe recebem das mãos. (Namora, $2018: 22$ )

Incarcéré dans les profondeurs de la terre, comme s'il creusait progressivement sa propre tombe, le mineur (aussi bien celui de Germinal que celui de Minas de San Francisco) perd à la fois sa santé et ses illusions dans la mine, alors qu'il se bat pour de meilleures conditions de vie et plus de justice. C'est le cas de Ti Cardo qui incarne la lutte quotidienne des paysans, présageant la fin terrible de la mine :

Uma praga passou ali, pela terra, e deixou-a assolada. Dói sentir essa desolação : dói a quem, desde moço, viu dos regos ensopados de chuva rebentarem searas com o loiro virgem dos trigos. A mina é uma enxurrada insaciável. Por onde passa, ficam destroços e desolações (...). Desse lugar, pode abranger a imensidão da mina, desafiá-la, gritar-lhe que ele nunca será vencido pela adulação do volfro. Ti Cardo é velho e pressente o futuro. Uma praga virá sepultar essa invasão diabólica, corroendo as vísceras das máquinas, decapitando, os tentáculos da cobiça. A praga virá ! É uma profecia. Sinais miúdos já a prenunciam, insidiosamente, deixando cicatrizes que os olhos cegos de San Francisco não suspeitam. (Namora, 2018 : 97)

Le leitmotiv du voyage aux profondeurs de la mine permet d'établir une espèce de topographie de la mine où les hommes sont bestialisés. Le lecteur assiste aux conséquences néfastes de l'euphorie du wolfram dans le paysage et chez l'homme. Cet Eldorado d'une vie meilleure ne sera en réalité qu'une illusion dans la mesure où les conditions de vie du mineur/paysan ne s'amélioreront pas. 
24 Passons maintenant à notre deuxième volet qui sera axé sur les implications idéologiques de ces voyages au centre de l'homme en tant que membre d'une communauté.

\section{Voyage et Idéologie}

Zola et Namora ont choisi l'espace de la mine, l'un des endroits les plus rudes et les plus difficiles où l'homme puisse travailler pour brosser un portrait fidèle de la mine, des mineurs et de leurs conflits sociaux. Namora transforme San Francisco en ce que Chalendar désigne comme « um microcosmos onde as leis do capitalismo campeiam e organizam com rigor implacável» (1979: 61). En ce qui concerne le discours idéologique du néoréalisme portugais, Carlos Reis met d'ailleurs en relief «cenários geográficos típicos e propícios à exposição dos conflitos sociais, ainda à selecção de personagens e caracteres significativos em termos de representatividade ideológica » (Reis, $1983: 534$ ).

C'est précisément ce qui se passe dans Minas de San Francisco où l'action a lieu dans les mines. En fait, le scénario de la mine montre les souffrances du peuple portugais (anciens agriculteurs et travailleurs) opprimé et exploité à la recherche d'une vie meilleure ainsi que les conflits sociaux et les luttes à mener pour avoir une vie plus digne. Le lecteur assiste ainsi à la métamorphose de l'ancien monde rural « no estranho mundo novo da mina » (Namora, 2018:51) avec une industrialisation de la campagne et un déplacement progressif des agriculteurs vers la mine.

27 Né à la campagne, le thème rural, ou plutôt, selon Óscar Lopes «a suite de cenas rurais» (1957: 5) traverse de nombreuses œuvres de Fernando Namora qui appartiennent au "cycle rural $»^{6}$ où Minas de San Francisco s'insère. Comme l'auteur l'affirme dans une interview de Encontros: "Os meus livros traçam com fidelidade a jornada do homem. Tenho escrito, enfim, o que vou experimentando " (Namora, 1981 : 29). En fait, Fernando Namora connaissait bien la géographie humaine qui parcourt ses livres, comme il l'a expliqué dans l'interview à Encontros :

As minhas origens são campesinas e entre os aldeões e a gente da vila passei a infância e largos períodos da adolescência. Esse clima humano foi a minha matriz. Podemos correr o mundo, arrancar a pele e mudá-la mil vezes, que a infância lá está como uma referência inapagável. (Namora,1981 : 153)

Or, ce voyage au centre de l'homme apparait forcément associé au paysage, au chemin de la transformation sociale, aux deux mines décrites et au soulèvement des mineurs. En réalité, la mine permet d'exposer les conflits sociaux, de mettre en scène les mauvaises conditions de travail, l'injustice, l'affrontement social, de dénoncer l'oppression et l'exploitation des mineurs. Ainsi, Étienne devient l'un des meilleurs herscheurs de la fosse et rêve de justice sociale, d'une révolution ouvrière, d'égalité et de fraternité entre tous les hommes. C'est pourquoi, il décide de créer une section de l'Internationale à Montsou et de créer une caisse de prévoyance pour faire face à une grève. Le discours d'Étienne sert justement la dimension idéologique du roman, incitant à un collectivisme radical. Il essaie de soulever les mineurs en dressant un tableau mythique, prophétique et grandiose de la lutte des classes. Centré sur un moment de l'histoire économique et sociale du $\mathrm{xIX}^{\mathrm{e}}$ siècle, le roman évoque le mouvement ouvrier avec la constitution des syndicats, les grèves et la naissance de la conscience des classes. 

conditions de vie des mineurs à celles des bourgeois, montrant l'opposition entre le capital et le travail. Les contrastes sociaux s'imposent entre l'espace bourgeois et l'espace des mineurs, étant véhiculés par l'évocation des odeurs et par la précision des notations olfactives. Le lecteur assiste ainsi à une déclinaison des espaces au long du roman : il y a ainsi l'espace où on a froid et celui où on a chaud; l'espace réduit et misérable et l'espace protégé et luxueux; l'espace où règne une odeur de brioche fumante et $\mathrm{du}$ chocolat chaud: "Cela sentait bon la nourriture. Des provisions débordaient des râteliers et des armoires (...). Le chocolat fumait dans les bols » (Zola, $1978: 122,128)$. Mais il y a aussi l'espace où règne une odeur d'oignon et un buffet vide sans provisions. En outre, il y a ceux qui sont trop bien nourris et ceux qui ont faim comme la famille Maheu et ses sept enfants-mineurs maigres, malades et mal nourris qui n'ont jamais pu profiter de leur enfance.

31 Construite en opposition, la description de la scène du lever de Cécile à la Piolaine chez les Hennebeau témoigne justement de cette lutte des classes sociales dans la mesure où elle contraste fortement avec le lever de Catherine dans le logis des Maheu. Tandis que Cécile est « trop saine, trop bien portante (...) avec une chair superbe, une fraîcheur de lait » (Zola, 1978: 123) et qu'elle peut bénéficier d'un sommeil paresseux : «elle a les yeux gonflés de sommeil» (Zola, 1978: 127); Catherine est mal nourrie, elle a «les pieds bleuis comme tatoués de charbon, et des bras délicats, dont la blancheur de lait tranchait sur le teint blême du visage » (Zola, 1978: 61) et elle se lève en pleine nuit pour travailler à la mine.

Cette conscience des classes sociales se manifeste aussi dans Minas de San Francisco. Dans le cas de Namora, cette lutte des classes est associée à la mission du groupe néoréaliste comme l'affirme Mário Sacramento: "A luta de classes era e é a luta mesmo, nós limitámo-nos a consciencializá-la» (Sacramento, s/d: 84). Selon Urbano Tavares Rodrigues, "a ideologia (...) do grupo social neo-realista, criador da vida intelectual e cultural da década de quarenta, manifesta-se não na consciência real das figuras, mas através delas » (1981b) : 98). En effet, la description du monde extérieur est étroitement liée à celle des hommes et c'est l'expression caustique de la réalité sociale qui montre au lecteur l'intimité d'une conscience sociale. C'est le cas, par exemple, de João Simão qui économise pour s'acheter un lopin de terre et qui accompagne la progressive dégradation de la mine. Alors que Quirino - autrefois pauvre, sans argent et humilié par opposition à l'argent, à la culture et au pouvoir des fonctionnaires de la mairie, des médecins et des propriétaires - est devenu quelqu'un grâce à la mine et à ses hommes : «E ele é um dos reis desse mundo (...) « Ele jurou um dia ser alguém no mundo novo da mina » (Namora, $2018: 51$ ).

Ce voyage à la mine permet au lecteur de prendre conscience de la vie de l'homme privé de liberté, de dignité et du besoin d'intervenir dans une société opprimée et déséquilibrée. La division de l'espace se manifeste entre les champs et la mine qui semblent s'affronter : "Ali terminam as árvores e o mato : os campos e a mina ficam a 
encarar-se ameaçadoramente, dois lutadores antes do assalto " (Namora,2018: 203), mais aussi entre l'espace du dessus et celui du dessous. Namora accompagne ainsi la douloureuse métamorphose des êtres humains qui transportent en eux le paysage des champs verdoyants. Ces êtres dont les mains s'adaptent avec violence à un nouveau code de gestes industriels dans une société aux classes antagoniques où les ressources continuent à appartenir à ceux qui commandent :

É gente nova que dirige San Francisco. Os estrangeiros, quando compraram a mina a um dono de ocasião - 'o Sequeira do minério'. (...) 'Uns fedelhos', dizem os mineiros coçados de serem conduzidos por gente sem barba na cara. Esses mineiros são homens que conheceram décadas de experiência no amanho das terras, tendo em cada ano uma coisa nova para aprender ; são homens que confundem sabedoria com mãos calejadas. Custa-lhes sujeitarem-se às ordens de quem só agora começa a desvendar o segredo das entranhas da terra. (Namora, $2018: 33$ )

Il y a ainsi plusieurs points de vue sur le paysage de la mine : celui du capital (le chef de la mine l'ingénieur Garcia et Almeida) et celui du travail que les mineurs personnifient. Le paysage peut ainsi assumer l'expression de propriété et d'exploitation de l'homme par l'homme.

En somme, c'est à partir de ces deux voyages au bout de la mine, qui se constituent comme un moyen d'accès privilégié au monde où s'entrelacent la narration et la description, qu'Émile Zola et Fernando Namora brossent une peinture des paysages, de l'homme et de la société. Cette dernière se manifeste à travers les descriptions riches qu'ils font de la société et de l'espace qui symbolisent aussi la lutte des classes. Ces deux voyages orientent ainsi le lecteur vers une réflexion sur la condition humaine grâce aux fresques humaines dont les deux œuvres se font les porte-voix. Truffées de scènes émouvantes, de descriptions des lieux, les deux romans sont imprégnés d'une approche sociale, cherchant à sauver la dignité humaine et d'autres valeurs immanentes à l'homme comme la liberté, la solidarité, la fraternité et le désir d'appartenance.

En décrivant les voyages et les actions des populations rurales, les deux auteurs concrétisent une intention sociale: dénoncer l'oppression et l'aliénation des plus démunis de la société. Tous deux choisissent comme protagoniste le peuple et ses luttes, séduisant ainsi le lecteur grâce à l'espoir en un monde plus humain et plus juste.

Germinal et Minas de San Francisco prônent en fait un message de vérité et de justice qui a fortement contribué à la prise de conscience du besoin d'une transformation sociale. Le peuple est vu comme une force souterraine dont le narrateur prédit l'avènement prochain et qui fera "bientôt éclater la terre " (Zola, 1978: 347). Nous sommes face à des forces humaines qui sont associées aux forces naturelles: les mineurs dans leur « uniformité terreuse » (Zola, 1978: 409) se confondent avec la terre. Les ravages de la misère et de la faim engendrent la violence des mineurs qui s'inscrit ainsi dans l'histoire des grandes luttes humaines pour la conquête du droit à l'égalité, à la dignité et à la recherche d'un avenir plus juste. Les deux romans se terminent d'ailleurs par l'acceptation des luttes à venir contre les multiples visages de la terreur, laissant entrevoir la promesse d'un monde plus juste tout en présentant les transformations sociales comme inévitables. 


\section{BIBLIOGRAPHIE}

ADAM, Jean-Michel (1989). Le texte descriptif. Paris : Nathan Université.

BAKHTINE, Mikhaill (1987). Esthétique et théorie du roman. Paris: Gallimard, “Tel.”

BASILIO, Kelly (1993). Le Mécanique et le vivant. La métonymie chez Zola. Genève : Droz.

BECKER, Colette (1998). Lire le Réalisme et le Naturalisme. Paris : éd. Dunond.

BECKER, Colette (1990). Zola en toutes Lettres. Paris : Bordas.

BECKER, Colette (1984). Germinal. Paris : PUF, « Que Sais-je ?».

BECKER, Colette, Gourdin-Serevenière Gina, Lavielle Véronique (1993). Dictionnaire d'Émile Zola.

Paris : éd. Laffont, « Bouquins ».

CHALENDAR, Pierrette e Gérard (1979). Temas e Estruturas na obra de Fernando Namora. Lisboa :

Moraes.

FONTANIER, Pierre [1968] (2009). Figures du discours (rééd.). Paris : Flammarion « Champs

Classiques », Préface de Gérard Genette.

HAMON, Philippe (1981). Introduction à l'analyse du texte descriptif. Paris : Hachette.

LEFEBVRe, Henri [1974] (2000). Production de l'espace (rééd.). Paris : Éditions Anthropos, « Société et urbanisme ".

LOPES, Óscar (1957). « Fernando Namora : Ensaio Crítico seguido de um inquérito ao autor

criticado », Separata da Revista Lusíadas, no 10, Porto, vol. III, pp. 3-10.

MITTERAND, Henri (2001). Zola II. L’Homme de ‘Germinal' (1871-1893). Paris : Editions Fayard.

MITERRAND, Henri (1990). «Chronotopies : la route et la mine », Zola, l'histoire de la fiction, Paris,

PUF, « Que Sais-je ?», pp. 179-198.

MITERRAND, Henri (1986). Zola et le Naturalisme. Paris : PUF, « Que Sais-je?».

MONTEIRO, Adolfo Casais (1950). o Romance e os seus Problemas. Lisboa : Livraria Editora da Casa do

Estudante do Brasil, "Biblioteca de Cultura Contemporânea.

NAMORA, Fernando (2018). Minas de San Francisco. Lisboa : Editorial Caminho, $1^{\mathrm{a}} \mathrm{Ed}$.

NAMORA, Fernando (1990). «Prefácio » a Casa da Malta. Mem Martins : Europa-América.

NAMORA, Fernando (1981). Encontros. Amadora : Bertrand.

REIS, Carlos (2011). «O Neorrealismo como Breve Memória », Jornal de Letras, Artes e Ideias, Ano

XXXI, $\mathrm{n}^{\mathrm{o}}$ 1066, pp. 6-7.

REIS, Carlos (2005). História Crítica da Literatura Portuguesa Volume X - Do Neo-Realismo ao PostModernismo. Lisboa : E. Verbo.

REIS, Carlos (1999). « Fernando Namora in AAVV 80 anos de Fernando Namora ». Oeiras : Câmara Municipal de Oeiras.

REIS, Carlos (1983). o Discurso Ideológico do Neorrealismo Português. Coimbra : Almedina.

REIS, Carlos (1981). « Apresentação Crítica », Textos Teóricos do Neo-Realismo Português. Lisboa : Seara Nova. 
RODRIGUES, Urbano Tavares (1981a). «Prefácio » a Um Novo Olhar sobre o Neo-Realismo. Lisboa : Moraes Editora.

RODRIGUES, Urbano Tavares (1981b). « Análise da obra de Fernando Namora a partir de Retalhos da Vida de um Médico e $O$ Trigo e o Joio », Um Novo Olhar sobre o Neo-Realismo. Lisboa : Moraes Editores. SACRAMENTO, Mário (1968). Há uma Estética Neo-Realismo. Lisboa : Dom Quixote.

SACRAMENTO, Mário (1967). Fernando Namora. Lisboa : Arcádia.

SACRAMENTo, Mário, s/d, Ensaios de Domingo III. Lisboa : Vega.

SAYEGH, Maria (2019). « La représentation des espaces et des paysages industriels dans Mes notes sur Anzin : réalité ou mythe? ", Landscapes of Industry : Zola and the Industrial Realities of his Time (Espaces et Paysages Industriels: Zola et les réalités sociales de son époque), in Valerie Minogue and Patrick Pollard (eds.). London : The Émile Zola Society London, pp. 73-84.

SESSAU, Claude (1989). Émile Zola, le réalisme symbolique. Paris : Éd. José Corti.

SICARD, Monique (2019). « Germinal : les facettes multiples du paysage », Landscapes of Industry :Zola and the Industrial Realities of his Time (Espaces et Paysages Industriels: Zola et les Réalités Sociales de son Époque), Valerie Minogue and Patrick Pollard (eds.). London : The Émile Zola Society London, pp. 33-48.

STONE, Shiloh (2019). « Le romancier mineur :Mining Novels in Nineteenth-Century France », Landscapes of Industry : Zola and the Industrial Realities of his Time (Espaces et Paysages Industriels : Zola et les Réalités Sociales de son Époque), Valerie Minogue and Patrick Pollard (eds.). London: The Émile Zola Society London, pp. 185-194.

TORGA, Miguel (1941). « Minério », Montanha. Coimbra : Atlântida.

zoLA, Émile (1978). Germinal. Paris: Gallimard, “Folio".

\section{NOTES}

1. Les différentes espèces dont parle Fontanier sont les suivantes: "la topographie, la chronographie, la prosopographie, l'éthopée, le portrait, le parallèle, le tableau” (1968: 422 ; $424-425$; 428-431).

2. C'est nous qui soulignons tous les italiques des extraits d'Émile Zola.

3. Comme l'a souligné Adolfo Casais Monteiro, à propos de Minas de San Francisco, «Este romance afirma-se como uma pintura suficientemente viva e densa para se ler dum fôlego - e eis virtude não pequena, sabendo-se como a pintura de vidas 'ao rés da terra', instintivas, primitivas, resulta geralmente duma monotonia insuportável» (1950 : 311). Dès l'incipit, nous pouvons remarquer ce que Adolfo Casais Monteiro considère "o pendor lírico [que] raramente o trai e, pelo contrário, conta muito para dar a todo o livro a necessária unidade de tom” (1950:311).

4. Le wolfram est le principal minerai de tungstène aussi connu comme " ouro preto ", un métal rare et précieux pour l'industrie. Le wolfram faisait aussi partie du catalogue de l'Exposition Industrielle Portugaise de 1888. Le Portugal a d'ailleurs été l'un des plus grands producteurs européens de wolfram étant parmi les dix premiers au niveau mondial.

5. C'est nous qui soulignons tous les italiques des extraits de Fernando Namora.

6. Du cycle rural font partie les oeuvres suivantes : Terra (1941); Casa da Malta (1945) ; Minas de San Francisco (1946) ; Retalhos da Vida de um Médico (1949) ; A Noite e a Madrugada (1950) e O Trigo e o Joio (1954). 


\section{RÉSUMÉS}

Cet article esquisse une réflexion comparative axée sur deux voyage(s) aux profondeurs de la mine à partir de l'analyse de Germinal (1885) d'Émile Zola (1840-1902) et de Minas de San Francisco (1946) de Fernando Namora (1919-1989) dont on a fêté le centenaire de la naissance en 2019. Notre hypothèse de lecture vise à analyser ce voyage au centre de la terre et de l'homme à partir de la topographie de la mine et de ses implications idéologiques dont les deux romans se font le porte-voix.

This article proposes a comparative study between two journey(s) to the depths of the mine, with an analysis of Germinal (1885) by French writer Émile Zola (1840-1902) and Minas de San Francisco (1946) by Portuguese writer Fernando Namora (1919-1989) whose 100th anniversary of his birth was just celebrated in 2019. This article will analyze the journey to the center of the Earth and the Human from the topography of the mine and its ideological implications, of which both novels voice in very different ways.

\section{INDEX}

Mots-clés : voyage, mine, idéologie, Zola (Émile), Namora (Fernando)

Keywords : travel, mines, ideology, Zola (Émile), Namora (Fernando)

\section{AUTEUR}

\section{ODETE JUBILADO}

Universidade de Évora- CEL

jubilado[at]uevora.pt 